\title{
ON WEIGHTED FOURIER INEQUALITIES - SOME NEW SCALES OF EQUIVALENT CONDITIONS
}

\author{
Alois Kufner, LARs-Erik Persson and Natasha SAmKo
}

\begin{abstract}
For Lebesgue spaces on $\mathbb{R}^{n}$, we study two-weight $p \rightarrow q$-inequalities for Fourier transform. Some sufficient conditions on weights for such inequalities are known for special ranges of parameters $p$ and $q$. In the same ranges of parameters we show, that in every case each of those conditions can be replaced by infinitely many conditions, even by continuous scales of conditions. We also derive some new such characterizations concerning the Fourier transform in weighted Lorentz spaces.
\end{abstract}

Mathematics subject classification (2020): 26D15, 42A38, 46E30.

Keywords and phrases: Fourier transform, weighted inequalities, scales of conditions, Fourier inequalities, Hardy-type inequalities, weighted Lebesgue spaces, weighted Lorentz spaces, continuity of the Fourier operator.

\section{REFERENCES}

[1] J. J Benedetto AND H. P. Heinig, Weighted Fourier inequalities: new proofs and generalizations, J. Fourier Anal. Appl., 9 (1): 1-37, 2003.

[2] J. Bergh AND J. LöFström, Interpolation Spaces - An introduction, Springer-Verlag, BerlinHeidelberg-New York, 1976.

[3] J. S. BRadley, Hardy inequalities with mixed norms, Canad. Math. Bull., 21 (4): 405-408, 1978.

[4] A. Gogatishvili, A. Kufner, And L. E. Persson, Some new scales of characterizations of Hardy's inequality, Proc. Est. Acad. Sci., 59 (1): 7-18, 2010.

[5] A. Gogatishvili, A. Kufner, L. E. Persson, And A. Wedestig, An equivalence theorem for integral conditions related to Hardy's inequality, Real Anal. Exchange, 29 (2): 867-880, 2003/04.

[6] A. Kufner And K. Kuliev, Some characterizing conditions for the Hardy inequality, Eurasian Math. J., 1 (2): 86-98, 2010.

[7] A. Kufner, L. E. Persson, ANd N. SAmko, Some new scales of weight characterizations of Hardy-type inequalities, Operator theory, pseudo-differential equations, and mathematical physics, Oper. Theory Adv. Appl., 228: 261-274, 2013.

[8] A. Kufner, L. E. Persson, And N. Samko, Weighted Inequalities of Hardy Type, World Scientific, New Jersey, 2017, second edition.

[9] G. G. LoRentZ, On the theory of spaces $\Lambda$, Pacific J. Math., 1: 411-429, 1951.

[10] V. MAZ'JA, Sobolev spaces, Springer Series in Soviet Mathematics, Springer-Verlag, Berlin, 1985. Translated from Russian by T. O. Shaposhnikova.

[11] B. Muckenhoupt, Hardy's inequality with weights, Studia Math., 44: 31-38, 1972. Collection of articles honoring the completion by Antoni Zygmund of 50 years of scientific activity, I.

[12] L. E. PERSSON AND V. STEPANOV, Weighted integral inequalities with the geometric mean operator, J. Inequal. Appl., 7 (5): 727-746, 2002.

[13] L. E. Persson And V. Stepanov, Some scales of equivalent weight characterizations of Hardy's inequality: the case $q<p$, Math. Inequal. Appl., 10 (2): 267-279, 2007.

[14] J. Rastegari And G. Sinnamon, Weighted Fourier inequalities via rearrangement, J. Fourier Anal. Appl., 24: 1225-1248, 2018.

[15] E. SAWYER, Boundedness of classical operators on classical Lorentz spaces, Studia Math., 96 (2): $145-158,1990$. 
[16] G. Sinnamon And V. Stepanov, The weighted Hardy inequality: new proofs and the case $p=1$, J. London Math. Soc. (2), 54 (1): 89-101, 1996. 